\title{
PROGRAMAS UNIVERSITARIOS PARA MAYORES. LA EDUCACIÓN PARA TODA LA VIDA: UNA EXPERIENCIA EN LA UNIVERSIDAD DE LAS PALMAS DE GRAN CANARIA
}

\author{
$M^{\mathrm{a}}$ AUXILIADORA GONZÁLEZ BUENO \\ Profesora Titular de Trabajo Social y Servicios Sociales. \\ Universidad de Las Palmas de Gran Canaria.
}

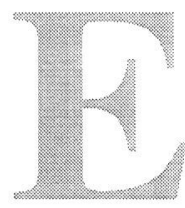

1 presente artículo intenta dar a conocer una experiencia de intervención socio-educativa que pone en marcha la Universidad de Las Palmas de Gran Canaria y que se enmarca dentro de la educación para toda la vida, como medida de protección social y atención a la tercera edad del nuevo milenio. Una experiencia de intervención socio-educativa desde el nivel superior de la enseñanza, como una forma de acercamiento de la Universidad a la realidad social.

Jacques Delors $(1999)^{1}$ manifiesta que frente a los numerosos desafíos del porvenir, la educación constituye un instrumento indispensable para que la humanidad pueda progresar hacia los ideales de paz, libertad y justicia social, como una vía al servicio del desarrollo humano más armonioso, más genuino, para hacer retroceder la pobreza, la exclusión, las incompetencias, las opresiones, las guerras, etc.

Este último cuarto de siglo ha estado marcado por notables descubrimientos y progresos científicos, muchos países han salido del subdesarrollo, el nivel de vida ha continuado su progresión con ritmos muy diferentes según los países. Y sin embargo, un sentimiento de desencanto parece dominar y contrasta con las esperanzas nacidas inmediatamente después de la última guerra mundial.

Ha quedado de manifiesto que el crecimiento económico a ultranza

1 VV.AA (1996): La educación encierra un tesoro. Informe a la Unesco de la Comisión Internacional sobre educación para el siglo XXI, presidida por Jacques Delors. Madrid. Santillana. Unesco. 
no se puede considerar ya el camino más fácil hacia la conciliación del progreso material y la equidad, el respeto de la condición humana y del capital natural que debemos transmitir en buenas condiciones a las generaciones futuras.

Todo convida entonces a revalorizar los aspectos éticos y culturales de la educación, atendiendo las misiones que debe cumplir la educación al servicio del desarrollo económico y social. Un sistema más flexible que permita la diversidad de estudios. La imaginación humana, precisamente para crear esta sociedad, debe adelantarse a los progresos tecnológicos si queremos evitar que se agraven el desempleo y la exclusión social o las desigualdades en el desarrollo.

\section{CONSIDERACIONES GENERALES DEL INFORME A LA UNESCO DE LA COMISIÓN INTERNACIONAL SOBRE LA EDUCACIÓN PARA EL SIGLO XXI}

La educación ocupa un lugar cada vez mayor en la vida de los individuos a medida que aumenta su función en la dinámica de las sociedades modernas. La división tradicional de la existencia en periodos claramente separados ha dejado de corresponder a las realidades de la vida contemporánea y se ajusta aún menos a los imperativos del futuro. Nadie puede hoy esperar que el acervo inicial de conocimientos constituido en la juventud le baste para toda la vida, pues la rápida evolución del mundo exige la actualización permanente del saber. Por otra parte, el acortamiento del periodo de actividad profesional, la disminución del volumen total de horas de trabajo remuneradas y la prolongación de la vida después de la jubilación aumentan el tiempo disponible para otras actividades.

Para organizar este proceso hay que dejar de considerar que las diversas formas de enseñanza y aprendizaje son independientes y, en cierta manera, imbricadas, si no concurrentes y, en cambio, tratar de realzar el carácter complementario de los ámbitos y los periodos de la educación moderna.

En primer lugar, como ya se ha indicado, la transformación del proceso de producción en aras de una mayor competitividad ha determinado que los saberes y las técnicas de cada individuo, adquiridos durante la formación inicial, pierdan rápidamente vigencia y se acentúe la necesidad de desarrollar la capacitación profesional permanente. La formación permanente responde en gran medida a un imperativo de orden económico. Por otra parte, brinda a los individuos la oportunidad de actualizar sus conocimientos y vislumbrar posibilidades de ascenso. 
Según la Comisión debe imponerse el concepto de educación durante toda la vida con sus ventajas de flexibilidad, diversidad y accesibilidad en el tiempo y el espacio. Debe ser una estructuración continua de la persona, de su conocimiento y sus aptitudes, pero también de su facultad de juicio y acción. Debe permitirle tomar conciencia de sí misma y de su medio ambiente e invitarla a desempeñar su función social en el trabajo y la ciudad. No es menos cierto que para poder utilizar bien ese potencial la persona debe poseer todos los elementos de una educación básica de calidad.

Para ello, nada puede reemplazar al sistema formal de educación en que cada uno se inicia en las materias del conocimiento en sus diversas formas.

En efecto, la Comisión piensa en una educación que genere y sea la base de este espíritu nuevo, lo que no quiere decir que haya descuidado los otros tres pilares de la educación que, de alguna forma, proporcionan los elementos básicos para aprender a vivir juntos.

La educación a lo largo de la vida se basa en cuatro pilares:

- Aprender a conocer, combinando una cultura general suficientemente amplia con la posibilidad de profundizar los conocimientos en un pequeño número de materias. Lo que supone, además, aprender a aprender para poder aprovechar las posibilidades que ofrece la educación a lo largo de la vida.

- Aprender a hacer a fin de adquirir no sólo una cualificación profesional sino, más generalmente, una competencia que capacite al individuo para hacer frente a gran número de situaciones y a trabajar en equipo. Pero, también, aprender a hacer en el marco de las distintas experiencias sociales o de trabajo que se ofrecen a los jóvenes y adolescentes, bien espontáneamente a causa del contexto social o nacional, bien formalmente gracias al desarrollo de la enseñanza por alternancia.

- Aprender a vivir juntos desarrollando la comprensión del otro y la percepción de las formas de interdependencia, realizar proyectos comunes y prepararse para tratar los conflictos respetando los valores de pluralismo, comprensión mutua y paz.

- Aprender a ser para que florezca mejor la propia personalidad y se esté en condiciones de obrar con creciente capacidad de autonomía, de juicio y de responsabilidad personal. Con tal fin, no menospreciar en la educación ninguna de las posibilidades de cada individuo: memoria, razonamiento, sentido estético, capacidades físicas, aptitudes para comunicar.

Todo este proceso lleva aparejado un proceso de reforma en todos 
los ámbitos educativos, y es necesario ir respondiendo a los interrogantes que se plantean en cada uno de sus ámbitos. Desde la Universidad se puede contribuir a esta reforma diversificando su oferta:

- Como lugar de ciencia y fuente de conocimiento que llevan a la investigación teórica o aplicada, o a la formación de profesores.

- Como medio de adquirir calificaciones profesionales conforme a unos estudios universitarios y unos contenidos adaptados constantemente a las necesidades de la economía, en los que se aúnen los conocimientos teóricos y prácticos a un alto nivel.

- Como plataforma privilegiada de la educación durante toda la vida, al abrir sus puertas a los adultos que quieran reanudar los estudios, adaptar y enriquecer sus conocimientos, o satisfacer sus ansias de aprender en todos los ámbitos de la vida cultural.

- Como interlocutor privilegiado en una cooperación internacional que permita el intercambio de profesores y estudiantes, y facilite la difusión de la mejor enseñanza mediante cátedras internacionales.

La Comisión se ha hecho eco de otra utopía: la sociedad educativa basada en la adquisición, la actualización y el uso de los conocimientos. La educación debe permitir que todos puedan aprovechar esta información, recabarla, seleccionarla, ordenarla, manejarla y utilizarla. Por consiguiente, la educación tiene que adaptarse en todo momento a los cambios de la sociedad.

Ahora bien, la educación a lo largo de la vida, en el sentido que le da la Comisión, va aún más lejos. Debe dar a cada individuo la capacidad de dirigir su destino en un mundo en el que la aceleración del cambio, acompañada del fenómeno de mundialización, tiende a modificar la relación de hombres y mujeres con el espacio y el tiempo. La educación a lo largo de la vida ha de brindar a cada cual los medios para alcanzar un mejor equilibrio entre el trabajo y el aprendizaje, y para el ejercicio de una ciudadanía activa.

La educación a lo largo de la vida representa para el ser humano una construcción continua de sus conocimientos y aptitudes y de su facultad de juicio y acción. El saber, el «saber hacer», el «saber ser» y el «saber convivir» en sociedad constituyen los cuatro aspectos, íntimamente enlazados, de una misma realidad. Combina el conocimiento formal y no formal, el desarrollo de aptitudes innatas y la adquisición de nuevas competencias. Además de ser una experiencia singular de cada persona, es también la más compleja de las relaciones sociales, pues abarca a la vez los ámbitos cultural, laboral y cívico. La educación durante toda la vida se presenta como una de las llaves de este siglo XXI. Se trata de aprender a vivir juntos conociendo mejor a los demás, 


\section{LA FORMACIÓN DE MAYORES EN EL ÁMBITO UNIVER- SITARIO}

La mayor esperanza de vida, la disminución de la tasa de natalidad y la jubilación anticipada, entre otros factores, están aumentando de forma absoluta y relativa a otros colectivos sociales, el número de personas «mayores» en nuestra sociedad. Personas mayores jubiladas, que no viejos, que disfrutan de tiempo libre y que exigen más cuota de participación social y en la solución de sus propios problemas (Lemiex 1997). ${ }^{2}$

Según describen Guirao y Sánchez-Martínez $(1997)^{3}$ para el caso español, se trata de una masa social de personas que no pudieron embarcarse en unos estudios universitarios: «Estamos hablando de generaciones de españoles que tuvieron que sufrir las repercusiones de nuestra guerra y posguerra civil, en un momento de desarrollo de España en el que la educación universitaria era un coto reservado para una elite de afortunados. Ahora es un buen momento para invitar a estas personas a cumplir lo que para muchos fue sólo un sueño: asistir a la Universidad».

La atención educativa a los mayores ha ido evolucionando desde las primeras experiencias de enseñanza para mayores en Norteamérica (1927) que no eran más que reuniones organizadas de personas mayores; les sigue la formación de las personas que intervienen en el entorno a los mayores, hasta la creación, en 1973, de la primera Universidad de la Tercera Edad en Toulouse por el profesor Pierre Vellas. Asistimos a la tercera generación en el modelo de atención educativa a los mayores, la de la Orientación Gerontológica y que se caracteriza y diferencia de otras iniciativas en que las Universidades ofrecen programas de enseñanza, investigación y de servicio a la colectividad. ${ }^{4}$

Ante la puesta en marcha de un programa universitario de atención a la tercera edad se recomienda, que atendiendo a este modelo de

2 Lemiex, A. (1997). Los Programas Universitarios para Mayores Enseñanza e Investigación. Ministerio de Trabajo y Asuntos Sociales (IMSERSO), Madrid. 163 pags.

Guirao, M. y Sánchez-Martínez, M. (1997). Los programas universitarios para mayores en España. En: Los Programas Universitarios para Mayores. Enseñanza e Investigación (André Lemiex, Editor), pp. 145-153. Ministerio de Trabajo y Asuntos Sociales (IMSERSO), Madrid.

4 Al definir el programa como un servicio a la colectividad evitamos desviaciones, por otra parte bastante frecuentes en las Universidades, hacia formas educativas más relacionadas con la Educación Superior como la organización de master, proyectos de investigación, departamentos universitarios especializados que no permiten el acceso de los actores principales del problema. 
tercera generación, se oferte una intervención tripartita que abarque la enseñanza, la investigación y el servicio a la colectividad.

A. Con respecto a la enseñanza:

- Que las Universidades continúen ocupándose de la educación de adultos hasta alcanzar a las personas mayores.

- Que para hacer esto se desarrolle un certificado de formación general para adultos mayores, al igual que se ha hecho (por otras instancias) para el primer ciclo educativo.

- Que en la formación de maestros y en el segundo ciclo se prevean acciones encaminadas a favorecer posteriormente un mejor entendimiento de los escenarios alternativos, salud, vivienda, tiempo libre, jubilaciones y roles sociales relacionados con la edad.

B. Con respecto a la investigación:

- Que se estimule la producción de memorias de masters y tesis doctorales en el área de la tercera edad.

- Que se estimule la producción científica en el área de la tercera edad.

C. Con respecto a los servicios a la colectividad:

- Que sea cual sea la forma en que se haga (programa de formación, centro de estudios universitarios, proyectos de investigación, etc.) se haga como un servicio de orden educativo y social destinado a la atención a mayores.

- Que se favorezca el acercamiento a los individuos y los organismos del entorno.

En España, las ofertas de actividades de carácter educativo y cultural dirigidas a los adultos mayores son de tres tipos: a) la de agrupaciones y asociaciones de difusión cultural, de formación permanente o denominación semejante, b) aulas, agrupaciones y asociaciones de extensión universitaria y c) la que constituyen los programas universitarios para alumnos mayores que se realizan en las propias universidades.

La Universidad de Las Palmas de Gran Canaria se decanta por esta tercera opción sin discriminar las anteriores, puesto que las mismas no se contraponen ni desvirtúan entre sí, sino que tratan conjuntamente de ampliar el panorama de una oferta solidaria para que la persona mayor pueda elegir con más libertad la opción que mejor atienda sus aspiraciones, necesidades y posibilidades. 


\section{PROGRAMA DE MAYORES DE LA UNIVERSIDAD DE LAS PALMAS DE GRAN CANARIA "PERITIA ET DOCTRINA"}

El programa Peritia et Doctrina es el resultado del compromiso adquirido por el equipo rectoral de la ULPGC a través del vicerrectorado de estudiantes y la Dirección General de Servicios Sociales de la Consejería de Empleo y Asuntos Sociales del Gobierno de Canarias. Surge hace tres años, en el curso 1998/1999 y en favor de una mayor implicación social, la ULPGC asume el reto de crear una iniciativa encaminada a dar asistencia educativa y permitir el acceso a la Universidad a sectores de la población distintos al tradicional.

Se constituye como una alternativa al colectivo de "mayores" como medida de protección social y atención a la tercera edad del nuevo milenio. Una experiencia de intervención socio-educativa desde el nivel superior de la enseñanza, como una forma de acercamiento de la Universidad a la realidad social. El programa de formación de mayores se concibe, por tanto, como la contribución de las Universidades a la atención social solidaria que, además, forman y con ello aumentan el nivel cultural de la comunidad.

El programa asume todos aquellos argumentos que hablan en favor de una mayor implicación social de la Universidad. Y es, desde este principio, que se considera los programas de formación de mayores como la contribución de las Universidades a atención social solidaria que, además, forman y con ello aumentan el nivel cultural de la comunidad.

El nombre del proyecto surge de la idea de combinar la Peritia: «la experiencia y el saber que traen los mayores por formación previa y/o edad» y Doctrina «el conocimiento que aún pueden adquirir"5.

\subsection{Objetivos del programa:}

1. Ser un servicio a la colectividad.

2. Complementar la oferta de formación y participación social de los mayores de 55 años.

3. Formar sobre temas específicos de la edad.

4. Formar sobre temas que permitan mejor adaptación a los cambios sociales, culturales y tecnológicos.

5. Integrar a los mayores de 55 años en la comunidad universitaria: para que conozcan la (su) Universidad y que el contacto permita el entendimiento intergeneracional. 


\subsection{Estructura del programa}

El programa no da lugar a competencias profesionales pero tiene organización administrativa y académica similar a las de las titulaciones de la Universidad. El alumno debe inscribirse, matricularse, asistir a clases en los centros de la Universidad y, sobre todo, aplicarse y rendir para titular.

Se estructura en tres años académicos con un total de 600 horas. Los cursos se desarrollan en dos trimestres, más un mes del período de formación permanente. Los alumnos reciben un total de 200 horas por curso, de las que un $70 \%$ son clases teóricas y el resto actividades dirigidas, trabajos prácticos, visitas culturales, incluso actividades y cursos de extensión universitaria. Los alumnos cursarán al menos diez materias por curso, de las cuales seis son obligatorias y doce optativas.

Las materias obligatorias tienen contenidos relacionados con la edad, mientras que las materias optativas abordan eminentemente aspectos culturales, sociales y tecnológicos que permitan una mejor adaptación a la sociedad moderna.

Las asignaturas del programa responden a las grandes áreas de la

CUADRO 1: Organización del título y materias.

\begin{tabular}{|l|}
\hline OBLIGATORIAS \\
\hline PRIMER CURSO \\
\hline Psicología de la madurez \\
Sistemas de Protección Social en la Edad Adulta \\
Estrategias para el desarrollo personal \\
Historia del Arte \\
Lengua y Literatura \\
Historia de España \\
\hline SEGUNDO CURSO \\
\hline Habilidades sociales II \\
Biología Vegetal y Naturaleza Canaria \\
Las telecomunicaciones en el nuevo milenio \\
Ciencia y Sociedad una relación permanente \\
Introducción al Derecho \\
Contaminación \\
\hline TERCER CURSO \\
\hline Solidaridad, Asociacionismo y Voluntariado \\
Movimiento y mecánica corporal \\
Historia de Canarias II \\
Marco jurídico específico del mayor \\
Biología del Sistema Nervioso \\
Dilemas éticos \\
\hline
\end{tabular}

\begin{tabular}{|l|}
\hline OPTATIVAS \\
\hline Informática básica \\
Introducción a la Sociología \\
Taller de musicoterapia \\
Energías alternativas \\
La pervivencia del pensamiento \\
y de la cultura grecolatina en el \\
mundo actual \\
Nutrición y dietética \\
Accesibilidad, barreras arquitec- \\
tónicas, urbanísticas y de trans- \\
porte \\
La salud en la edad adulta \\
Riesgos y cuidados sanitarios en \\
las actividades de la vida diaria \\
Historia de la música \\
Internet y las nuevas comunica- \\
ciones \\
Relaciones humanas (interperso- \\
nal y grupal)
\end{tabular}


Universidad. De esta manera se abordan materias relacionadas con las disciplinas Humanístico-Lingüísticas, Científicas, Tecnológicas, de la Salud, del Deporte y del Derecho, entre otras. Los tres cursos se desarrollan en el área de San Cristóbal (nuevo centro destinado a dicha formación).

Fruto del alto interés mostrado y a demanda del alumnado, se ha generado una nueva oferta de formación más específica y a la que denominamos periodo de formación permanente, cuyo objetivo es ofrecer módulos de especialización en relación con las materias cursadas o temas de interés del momento (gestión de asociaciones en Canarias, acercamiento a las matemáticas en su año internacional, la inmigración en Canarias y su perspectiva social, etc:).

\subsection{Evaluación}

La evaluación intenta medir el grado de logro alcanzado por los estudiantes a lo largo del curso y la consecución o no de los objetivos planteados inicialmente. Asimismo, consideramos que la evaluación debe medir el rendimiento del estudiante de forma continua. La metodología a seguir durante el curso se centra en la participación e implicación activa de los alumnos en su proceso de aprendizaje.

Las clases compaginarán la aportación de conocimientos teóricos por parte del profesor/a con clases prácticas a través de los diferentes trabajos grupales que se puedan ir realizando de los diferentes temas tratados, como vía de profundización del alumno. Se exige la asistencia al $80 \%$ de las horas lectivas para obtener el diploma acreditativo del curso.

\subsection{Perfil de alumnado}

El programa cuenta con 200 alumnos matriculados en los tres cursos del programa. La edad media de los mismos se sitúa en torno a los 65,43 situando el rango de edad entre los 55 y los 87 años. Se trata de personas integradas en la comunidad y residentes en su mayoría en la ciudad de Las Palmas.

En relación a la variable sexo nos encontramos con una mayor presencia de mujeres, con un $62 \%$ frente a un $38 \%$ de hombres, donde el estado civil es variado, predominando las categorías casados y viudos.

El nivel de estudios y el tipo de profesión ejercida también es variado, existiendo una mayor proporción de personas con estudios primarios, seguido de los estudios medios, tales como maestros, enfermeras, etc. En relación a la actividad profesional abunda la formación profesional no reglada (técnicos en general y profesionales del sector servi- 
cios) y profesiones liberales (pequeños y medianos empresarios).

En relación a la asistencia nos encontramos con un alto índice de participación, con un $87 \%$ de alumnos que asisten regularmente y un $10 \%$ de ausencias justificadas. El abandono es bastante bajo, con un $3 \%$ de los matriculados.

\subsection{Líneas futuras de actuación}

Nuestras líneas futuras de actuación tendrán una doble vertiente: en primer lugar, y a nivel académico, seguir actuando en la consecución de los objetivos del programa, mejorando y potenciando las tres grandes áreas propuestas:

- Con respecto a la educación, seguir ampliando la optatividad de formación básica y los módulos de formación especializados.

- En relación a la investigación, continuar con el apoyo y fomento de líneas investigadoras "con" y "para" este colectivo.

- Y, en último lugar, y dentro del apartado de servicios a la comunidad, fomentar el asociacionismo de los mayores y su participación activa en la comunidad.

Por otra parte, y a nivel institucional, seguir consolidando el programa en el engranaje de la Universidad hasta lograr la plena integración del programa, así como la introducción del mismo en las redes nacionales e internacionales de formación de mayores.

\section{EN CONCLUSIÓN}

Como conclusiones generales a los programas de mayores en las Universidades españolas aportamos el trabajo conjunto en el V Encuentro Nacional de Programas Universitarios para Mayores, celebrado en Tenerife en abril de 2001. Entre las conclusiones alcanzadas se destacan:

\section{A) Acuerdos generales:}

- Constituir la comisión nacional de programas universitarios para mayores como órgano de coordinación entre los distintos programas universitarios. Dicha comisión tendrá, entre otras, la función de preparar el próximo encuentro nacional.

- Enfatizar la dimensión universitaria de los programas para mayores y, por tanto, destacar la formación científica y humanista de los mismos.

- Estimular al profesorado universitario de los programas para que impulsen investigaciones en el marco de estos programas con el fin 
de que permitan conocer con mayor profundidad sus diversos aspectos.

- Favorecer los procesos de evaluación de los programas como una manera de mejorar la calidad de los mismos.

- Actualizar el censo de programas y que éste se incluya en la guía de programas universitarios para mayores que edita el IMSERSO.

- Potenciar el intercambio con instituciones europeas similares a los programas para mayores a través de acciones que, como el Grundtvig, pueden posibilitar el intercambio de alumnado y profesorado de la Unión Europea.

- Favorecer el uso de las nuevas tecnologías desde los programas para mayores.

- Reconocer el carácter social de los programas para mayores y, en este sentido, solicitar la colaboración del IMSERSO y que esta institución esté abierta a la colaboración con las nuevas iniciativas que se presenten y a potenciar, en la medida de lo posible, el desarrollo de las ya existentes.

b) Acuerdo del alumnado

- Solicitar de las Universidades el reconocimiento oficial que posibilite, tras haber superado los tres cursos correspondientes, el acceso a la Universidad reglada sin otro requisito.

- Que el alumnado participe oficialmente en la decisión, tanto de las materias a impartir en las clases como del profesorado.

- Que se regulen los planes de estudio de forma que el primer ciclo conste de tres años como mínimo.

- Que exista en el órgano de gobierno de la Universidad la correspondiente presencia institucionalizada (con voz y voto) del alumnado mayor.

- Que el alumnado, una vez acabado el primer ciclo, tenga la posibilidad de asistir a cursos monográficos y/o diversificados.

c) Acuerdos de las asociaciones

- Necesidad de que se organicen asociaciones de alumnado mayor en aquellas Universidades donde no existan.

- Independencia de las asociaciones en sus propuestas y actos.

- Que las asociaciones tengan la oportunidad de federarse.

d) Acuerdos de la federación

- Necesidad de integración en la federación nacional de todas las asociaciones de programas universitarios.

- Formar parte en la formación y desarrollo de programas universitarios para mayores. 
- Integración en los órganos de gobierno de las Universidades y entidades colaboradoras con los programas.

En último lugar y aterrizando en la Universidad de Las Palmas de Gran Canaria, destacamos que, tras tres años de andadura, hemos comprobado cómo el programa "Peritia et Doctrina" no sólo se configura como un espacio académico donde un grupo de personas mayores se encuentran para mantener frescas y ágiles sus mentes, sino que por el contrario se ha configurado como un grupo de participación social ciudadana, surgiendo del mismo una asociación cultural de ocio y tiempo libre, además de lograr la participación e implicación de otros organismos y entidades socio-económicas en actividades de tipo educativo.

Podríamos afirmar que este tipo de programas comienzan a configurarse como nuevas medidas de protección social hacia un colectivo con necesidades sociales muy diversas y cambiantes, por lo cual es necesario arbitrar políticas sociales concretas que den soluciones al nuevo colectivo de mayores al que nos enfrentamos en el nuevo milenio, un colectivo con mucho más peso social y sobre todo más implicado en la sociedad a la que pertenece.

\section{BIBLIOGRAFÍA}

DE CASTRO, A. (1990): La Tercera Edad: Tiempo de ocio y cultura. Madrid. Narcea.

DOCUMENTACIÓN SOCIAL (1992): La Animación de los Mayores. Revista de Estudios Sociales y de Sociología Aplicada, nº 86.

GUIRAO, M. y SÁNCHEZ MARTÍNEZ, M. (1998): La oferta Gerontológica. Universidad de Granada. Editorial Universitaria.

KARLHEINZ, A. y MARIANNE HEGE (1997): Acción Socioeducativa: Modelos, métodos y técnicas. Madrid. Narcea.

LEMIEUX ANDRÉ (1997): Los programas Universitarios para mayores: Enseñanza e investigación. Bilbao. Imserso.

MARION L. BEAVER y DON A. MILLER (1998): La práctica clínica del Trabajo Social con las personas mayores. Barcelona. Paidós.

MEDINA FERNÁNDEZ, O. (1997): Modelos de educación de personas adultas. Universidad de Las Palmas de Gran Canaria. Roure.

VELÁZQUEZ y FERNANDEZ, C. (1998): Las Universidades de mayores una aventura hecha realidad. Universidad de Sevilla. Secretariado de Publicaciones.

VV.AA (1996): La educación encierra un tesoro. Informe a la Unesco de la Comisión Internacional sobre educación para el siglo XXI, presidida por Jacques Delors. Madrid. Santillana. Unesco. 